\title{
La Revue des catalogues
}

\section{Martine Mille}

\section{(2) OpenEdition}

\section{Journals}

Édition électronique

URL : http://journals.openedition.org/dht/1049

DOI : $10.4000 /$ dht. 1049

ISSN : 1775-4194

Éditeur :

Centre d'histoire des techniques et de l'environnement du Cnam (CDHTE-Cnam), Société des élèves du CDHTE-Cnam

Édition imprimée

Date de publication : 1 juin 2008

Pagination : 250

ISBN : 978-2-95-30779-7-9

ISSN : 0417-8726

\section{Référence électronique}

Martine Mille, "La Revue des catalogues », Documents pour l'histoire des techniques [En ligne], 15 | 7 er semestre 2008, mis en ligne le 21 octobre 2010, consulté le 24 septembre 2020. URL : http:// journals.openedition.org/dht/1049; DOI : https://doi.org/10.4000/dht.1049

Ce document a été généré automatiquement le 24 septembre 2020.

(c) Tous droits réservés 


\title{
La Revue des catalogues
}

\author{
Martine Mille
}

1 La revue Documents pour l'histoire des techniques propose d'ouvrir une rubrique de comptes rendus de catalogues d'expositions. En effet, ouvrages souvent luxueux, à la riche iconographie, les catalogues permettent de prolonger les expositions temporaires, elles-mêmes moyens aujourd'hui privilégiés d'animation et de diffusion culturelle à destination de publics multiples.

2 Au terme de l'exposition, quand les objets et les œuvres sont rendus aux collections permanentes, remis dans les réserves, restitués aux prêteurs, seul le catalogue permet de retracer le travail de recherche des équipes (souvent interdisciplinaires) de conservation et de restauration effectué pour la sélection, la documentation et la mise en valeur d'ensembles éphémères. Les bibliothèques nationales et les musées ont compris l'intérêt exceptionnel présenté par les catalogues, seul témoignage durable du travail réalisé sur un sujet sélectionné : depuis 2004, ce sont plus de 110000 livres d'exposition, plus de 23000 guides de musées que propose au chercheur le catalogue de la bibliothèque de l'Institut national d'histoire de l'art (INHA).

3 L'intérêt des catalogues d'exposition réside d'abord dans leur atout muséologique, la tendance actuelle étant de rendre compte de la dynamique de la scénographie, des codes de couleurs et de l'ambiance des salles autour des thématiques d'exposition. C'est le catalogue lui-même que les auteurs organisent bien souvent selon une scénographie «dans l'esprit » de l'exposition. Cet aspect mérite autant que le sujet de l'exposition une approche spécifique qui précise pour le lecteur la construction de la présentation temporaire de collections. La "revue des catalogues " proposée dans les Documents pour l'histoire des techniques vise à conserver la mémoire de ces expositions et à pointer les techniques de mise en scène ou de restauration des œuvres. Le catalogue est appréhendé comme objet et sujet de restitution de l'exposition.

4 En développant des thématiques à partir d'une exposition, les catalogues s'inscrivent aussi dans des enquêtes en cours en histoire des techniques. C'est dans la mesure où ils participent de questionnements à l'œuvre dans notre domaine qu'ils retiennent toute notre attention. Plus que l'actualité des expositions, c'est la réflexion sur les objets techniques et leurs images d'une part, sur la dynamique de recherche et ses liens avec 
l'analyse des artefacts d'autre part, qui justifie qu'une rubrique leur soit consacrée dans notre revue.

Qu'il nous suffise de citer certaines expositions récentes comme celles du Musée des arts et métiers, Benjamin Franklin, homme de science, homme du monde ou Visites dessinées dont nous proposons des comptes rendus dans ce numéro et le suivant. Nous y associons les arts décoratifs, avec l'exposition Du dessin au vitrail. Peintres et maîtres verriers du xixe siècle au nord de la France, présentée en 2006 au Palais des Beaux-Arts de Lille, et l'environnement, l'espace industriel urbain grâce à l'actuelle exposition Pays-âges au Musée d'histoire naturelle de Lille (également Musée industriel). Enfin les espaces culturels misant sur la diversité des publics peuvent par leurs catalogues se montrer d'un intérêt indéniable, tels les Rêves mécaniques autour de Léonard de Vinci à Rombas en Moselle. Rédigés avec méthode et rigueur, accompagnés d'articles de synthèse et d'une iconographie renseignant les collections, les catalogues constituent une source documentaire précieuse. Plus que la mémoire de l'exposition, ils s'imposent comme ouvrages de référence.

\section{AUTEUR}

MARTINE MILLE

CDHTE-Cnam 